\title{
Zur Lehre von der Tripperinfection beim Weibe.
}

\author{
Von
}

\section{Heinrich Fritsch,}

Docent in Falle.

Die Nöggerath'sche Schrift”) über die latente Gonorrhoe bei weiblichen Geschlecht hat wenig Kritik erfahren. Die Behauptungen des genannten Autors sind mit solcher Sicherheit. vorgetragen, dass ihre Unrichtigkeit nur an der Hand von Krankengeschichton bewiesen werden kann. Diese wiederum mussten mit besonderer Berücksichtigung der Nöggerath'schen Arbeit gesammelt werden, so dass es fast unmöglich war, ältere Beobachtungen zu verwerthen. Deshalb trete ich erst nach vier Jahren an die Oeffentlichkeit und hoffe jetzt über genügendes Material zu verfügen, um ein Urtheil fällen zu können.

Das Neue in Nöggerath's Abhandlung findet sich hauptsächlich in folgenden zwei Behauptungen concentrirt: Erstens soll der Tripper beim Manne unheilbar sein. Er besteht völlig latent und kann trotzdem noch nach Jahren z. B. nach 10 Jahren, (13. Krankengeschichte) der scheinbaren, vollständigen Heilung übertragen werden. Zweitens, soll nach gonorrhoiseher Infection beim. Weibe stets der ganze Tractus von der Vulva bis zum Abdominalende der Tuben erkrankt sein. Auch hier ist der Tripper unheilbax, und die von ihm abhängige Perimetritis wurde noch niemals geheilt.

Die Heilbarkeit des männlichen Trippers zu beweisen, muss ich den bezüglichen Specialisten iiberlassen, kann aber versichern, dass ich nicht wenige Ehemänner kenne, welche Kinder und ge-

1) Die latente Gonorrhoe im weiblichen Geschlecht; von Dr. Emil Nöggerath in New-York. Bonn bei Cohen, 1872. 
sunde Frauen besitzen, obwohl sie früher an manchem Tripper litten.

Was zunächst die Behauptung der völligen Latenz einer Krankheit anbelangt, so ist dies eine Contradictio in adjecto. Eine somatische Krankheit ist etwas Factisches, das Substrate hat, und nicht wie ein geheimnissvoller Fluch an dem Organe klebt. Fehlen alle handgreiflichen, sichtbaren oder aus den Folgen für das Organ selbst zu beweisenden Veränderungen, so fehlt auch eine Krankheit.

Aus vielen Nöggerath'schen lirankengeschichten geht aber hervor, dass es gar nicht nöthig ist, eine Latenz anzunehmen. Man weiss ja, wie lange Zeit die Gonorrhoe zur Heilung braucht. Jeder Arzt hat Fälle gesehen, wo der Patient sich für vollkommen geheilt hielt, während thatsächlich der Tripper als Eiter im Urin, als Verklebung der Harnröhre noch nachweisbar war. Es ist eine alltägliche Beobachtung, dass Patienten nach jedem Excess in Venere et Baccho einige Tage an deutlichem Tripper leiden, welcher ohne diese Excesse sich höchstens durch F'lecke in der Leibwäsche documentirte. Nun wird bekanntlich über Nichts in der Welt mehr gelogen, als über Tripper und Syphilis. Wer hier nicht vollkommener Skeptiker ist, erfährt die wunderbarsten Dinge. Selbst nach Einräumung des Factum, wird die Erzählung so verbrämt und verziert, dass der Fall als ein aussergewöhnliches, noch nie dagewesenes Unglück erscheint. Berichtet deshalb Nöggerath, dass die Heilung des Trippers erst vor 2 Monaten (Krankengeschichte 1), vor 3 Monaten (2, 3, 8, 10), oder vor „einiger Zeit", vor „einigen Monaten", vor „kurzer Zeit" erfolgt sei, so werden wir gewiss die völlige Latenz dieser Tripper bezweifeln dürfen.

Bedenkt man ferner, dass der schädliche, eine Recrudescirung des Nachtrippers verursachende Excess in Venere nach der Verheiratbung, selbstrerständlich ist, so verlieren diese Fälle an Beweiskraft für Nöggerath, und sind als Uebertragung eines immerhin frischen Trippergiftes anzusehen.

Mit der Behauptung von der Schädlichkeit einer derartigen Infection sagt uns aber Nöggerath nichts Neues. Schon ältere Schriftsteller haben dergleichen Beobachtungen gemacht, und Neuere haben es niemals geleugnet, dass Perimetritis in Folge einex Ansteckung mit Trippervirus entstehen könne.

Die zweite Behauptung, dass die gonorrhoische Erkrankung 
den gesammten Genitaltractus ergreife, will ich durch einige Krankengeschichten widerlegen. Seit Erscheinen der Nöggerath'schen Schrift suchte ich nach ähnlichen Fällen, und ich habe in dieser Zeit eine ziemliche Ánzahl von virulenten Scheidenkatarrhen bei Puellis publicis behandelt. Leider verschwinden derartige Patientinnen sofort aus der Bebandlung, wenn der Erfolg derselben in die Erscheinung tritt. Es ist deshalb ein solches Material nicht besonders geeignet zur Beschaffung werthvoller Krankengeschichten, und ich bin gezwungen mich auf die wenigen, vollständig beobachteten zu beschränken. Von diesen Krankengeschichten würde schon eine einzige genügen, die Nöggerath'schen Dogmen za Falle zu bringen.

1) Frau C. ist vor drei Monaten niedergekommen. Meine Hülfe wird wegen Brennen und Schmerzen an den Genitalien und beim Uriniren requirirt. Ich finde beide grossen Schamlippen erheblich geschwollen. Die Schambaare sind mit blutigem Eiter besudelt. Die fest aneinanderliegenden grossen Schamlippen sind vorn durch harte Krusten verklebt. Nach der Reinigung in einem Sitzbad, zeigen sich an der sehr schmerzhaften, hochrothen Innenfläche eine Anzahl kreisrunder flacher Geschwüre. Beim Druck anf den Damm fliesst viel Eiter aus der Vulya. Eine Exploration wurde wegen Schmerzhaftigkeit zunächst unterlassen. Die Therapie bestand in Sitzbädern, fortwährenden Umschlägen mit $2 \%$ Carbolsäurelösung und zweistündlicher Irrigation der Scheide mit derselben Lösung. An dritten Tage Specularuntersuchung. Die Röthung erstreckt sich bis an die Portio. Der Muttermund und Cervix sind aber nicht betheiligt, aus letzterer fliesst ganz wenig glasheller Schleim aus. Eine Affection an, oder um den Uterus lässt sich wegen des Mangels jeder Empfindlichkeit beim Bewegen des Uterns und der combinirten Untersuchung ansschliessen. Schon nach einer Woche tritt völlige Heilung ein.

Der Ehemann gab zu, noch jetzt an einem stark secernirenden Tripper zu leiden.

Ich beobachtete Patientin danach noch $3 / 4 \mathrm{Jabr}$. Sie blieb völlig gesund, ist jetzt im vierten Monat schwanger, und hat niemals die geringste Spur einer Perimetritis gehabt.

2) M. Puella publica, kommt wegen Fluor albus zu mir. Sie will vor 14 Tagen angesteckt sein und klagt über enormes Brennen in den Geschlechtstheilen, Schwellung der Vulva und Schmexzen beim Urinlassen. Die Inspection der Vulva zeigt eine rechtsseitige Entziündung der Bartholinischen Drüse, dem Durchbruch nahe. Die Hobe der Geschwulst ist schwarzbräunlich, die Schamhaare sind mit frischem und vertrocknetem, blutigen Eiter beschmutzt. Es bestehen die Symptome des Vaginismus. Druck auf die Harnröhre ist emptindlich. Nach dem Hemd zu urtheilen ist der Ausffuss sehr bedeutend. Der Abscess wird eröffnet, es fliesst bräunlicher, blutiger Eiter aus. (Zum 
Unterschied von den seltenen, chronischen Entzündungen der Bartholinischen Driuse, bei welchen man dünnen wässerigen Eiter, wie bei Dacryocystoblennorrhoe aus dem etwas erweiterten Ausführungsgange, ziemlich schmerzlos ausdrücken kann, ist bei den virulenten Entzü̈ndungen der Ausfiibrungsgang oft kaum aufzufinden, und der Eiter stets mit Blut vermischt.) Die Therapie war dieselbe wie im Falle 1. Die Scheide konnte schon axn zweiten Tage mit dem Speculum untersucht werden. Dabei zeigt sich, dass die Entzündung und Röthung blos die untere Hälfte der Vagina betrifft. Die Umgebung der Portio war auffallend blass. Cervicalkatarrh, sowie jede Schmerzhaftigkeit des Leibes fehlte. Patientin stellte sich nicht wieder vor.

Nach 6 Wochen werde ich gerufen. Patientin berichtet, dass schon nach einer Woche die ganze Affection geheilt sei. Jetzt ängstigten sie eine Menge über den hinteren Theil der Vulva und um den Anus sitzender, kleiner spitzer Condylome. Obwohl dieselben keinerlei Symptome machten, wurden sie doch in der Chloroformnarkose sämmtlich mit der Scheere abgeschnitten.

Nach wiederum einem Jahre bekam ich das inzwischen geborene Kind der Patientin in Behandlung. Es war ein höchst elender, sechswöchentlicher Säugling, der bald starb. Patientin war vollkommen gesund: Bei der Untersuchung fand ich den noch grossen Uterus völlig schmerzlos, frei beweglich. Patientin berichtete, dass sie schon am fünften Wochenbettstage ihr. Geschäft in einer Bierwirthschaft wieder aufgenommen habe.

3) Frau A., robuste Multipara vom Lande. Der Mann gesteht ein, dass er, als seine Frau hochschwanger gewesen sei, einen Tripper acquirirt habe. 19 Tage nach der Geburt habe er noch bestanden. Damals sei zuerst der Coitus wieder ausgeübt. Ihm sei eine Blutung bei seiner Frau gefolgt. Danach habe sich weisser Fluss eingestellt, und jetzt könne seine Frau wegen einer Geschwulst zwischen den Beinen das Bett nicht verlassen.

Bei der Untersuchung findet sich ein grosser Abseess in der rechten grossen Schamlippe. Derselbe wird durch Incision entleert. Der bedeutende, complicirende Vaginalkatarrh, wie in den andern. Fällen behandelt, heilt binnen einer Woche. Niemals Spur von einer Perimetritis. Nach 11 Monaten erfolgte eine glückliche Geburt ohne Wochenbettserkrankung. Heute $1^{3 / 4} \mathrm{Jahr}$ nach der Vaginitis ist die Frau völlig gesund.

Aus den vorstehenden Fällen ist also ersichtlich, dass die gonorrhoische Ansteckung eine Erkrankung hervorbringen kann, welche sich auf die Vulva und den zunächst über ihr liegenden Theil der Vagina beschränkt. Ferner lehren diese Fälle, dass unter Umständen die Heilung leicht ist. Ja ich behaupte, dass die Vaginitis virulenta, im Ganzen eine ungleich bessere Prognose giebt als die Urethritis beim Manne, und dies einfach deshalb, 
weil die Vagina leicht zugänglich ist, und die Secrete leicht zu entfernen sind.

Vor allem aber zeigen Fall 2 und 3, dass eine Infection mit Trippervirus für ein späteres Wochenbett keine so hochtragische Bedeutung hat, wie Nöggerath meint. Es wird sich dies einfach so verhalten, dass bei bestehender Perimetritis durch die mechanischen Einwirkungen dex Geburt ein Heftigerwerden der schon vorhandenen Krankheit eintritt.

Unschwer wäre es, Fälle zu sammeln, wo die gonorrhoische Erkrankung blos die Vulva betraf, als entzündlicher Vaginismus gedeutet und binnen kurzem bei antiphlogistischer Behandlung zur Heilung gebracht wurde. Solche Fälle sind so alltäglich, dass jeder Gynäkologe sich erinnern wird, sie gesehen zu haben.

Ich gehe nunmehr zu den Fällen über, bei welchen die gonorrhoische Affection sich der Cervix mitgetheilt hatte.

4) Frau P. Der Ehemann ist Kaufmann und leidet seit 12 Jahren an einem Tripper, welcher keineswegs latent ist. Alljährlich nach Reisen, und dem damit verbundenen, unregelmässigen Leben treten 2-3 Mal Reerudescirungen ein. Namentlich in der letzten Zeit war dem Secret viel Blut beigemischt, Beim Catheterisiren findet sich eine enge, höchst schmerzhafte, leicht blutende Stelle im hintern 'Theile der Harnröhre. Ein Specialist, welchem ich den Patienten zur Behandlung überwies, diagnosticirte Geschwüre der Urethralschleimhaut, und stellte den nunmehr sehr willigen und gewissenhaften Patienten doch erst in $3 / 4$ Jahrea her.

Die Patientin ist klein und colossal feist. Sie hat vor sieben Jahren ihr erstes Kind geboren und seitdem nicht wieder concipirt. Schon während der Schwangerschaft bestand ein starker Fluor, wurde aber aus Fureht vor Abort unbehandelt gelassen. Seit der Zeit dauert der Ausfluss fort.

Patientin hat, namentlich im Sommer, in allen Hautfalten, unter den Brüsten, am Nabel, unter den Armen und namentlich in der Nähe der Geschlechtstheile Erytheme und Eczeme. Die Innenfläche der Oberschenkel ist zum Theil aufgeätzt, zum Theil trocken, bräunlich, abschilfernd. Es finden sich einige Zellgewebsabscesse. $\mathrm{Zu}$ Zeiten sollen wochenlang immer neue, nicht sehr schmerahafte Abscesse entstehen. Die Vulva ist geröthet und schmerzhaft, die Urethralmündung hochroth und empfindlich. Die ganze Scheide ist aufgelockert und entzündet. Der äussere Muttermund, umgeben von der sehr dicken Portio, zeigt Erosionen. Sobald dieselben, vom Gegendruck der Scheidenwände befreit, im Speculum stehen, fangen sie an zu bluten. Die Innenfläche der Cervix ist mit viel diünnem Eiter und sehr schwer zu entfernendem Schleim bedeckt. Die combinirte Untersuchung zeigt den Uterus und seine Umgebung völlig schmerzlos. 
Die Portio wird sorgfältig gereinigt und dann gestichelt, bei letzterer Procedur entleeren sich vorher nicht gesehene Ovula Nabothi. Dann wurde die Portio in Acidum pyrolignosum gebadet, für die Nacht mit einem Tanninglycerinverband versehen und täglich vier Mal mit Carbolsolution irrigirt. Es tritt nach dieser Behandlung jedesmal erhebliche Besserung ein. Doch kehrt der Fluor nach 3-4 Wochen wieder, da die ausserhalb wohnende Patientin sich einer consequenten, lïngeren Behandlung nicht unterwerfen kann.

Niemals aber in den 8 Jahren, welche Patientin an dem Catarrh leidet, und in den 2 Jahren meiner Behandlung, trat auch nur die Spur einer perimetritischen Affection ein. Und somit ist der Beweis geliefert, dass ein auf Gonorrhoe zurückzubeziehender Catarrh der Cervix lange Zeit für sich bestehen kann.

5) Frau K. hat im Jahre 1855 von ihrem Mann eine Gonorrhoe acquirirt, welcher Umstand als Grund des Leidens bereitwillig genannt wird. Im vierten Monat der ersten Schwangerschaft in demselben Jahre abortirte Patientin. Seit dieser Zeit habe Patientin an Fluor, enormen Schmerzen beim Uriniren und fortwährendem Brennen gelitten. Lange Jahre habe ein Ausfluss bestanden. Nach der Fehlgeburt sei der Beischlaf so schmerzhaft gewesen, dass er niemals wieder ausgeübt wurde. Auch sei keine Neigung dazu vorhanden gewesen. Ich sah Patientin zuerst im Januar 1875, wo sie mich wegen klimacterischer Beschwerden consultirte. Das alte Leiden wurde blos beiläufig erwähnt. Bei der Untersuchung fand ich einen Zustand der Vagina, welchen ich weder selbst jemals gesehen noch beschrieben gefunden habe. Vom Introitus Vaginae an waren die Rugae so hypertrophirt, dass es unmöglich war mit dem Finger in die Vagina zu dringen. Die anämischen, keine Feuchtigkeit secernirenden Wucherungen sahen wie spitze Condylome aus, waren aber nicht zerklüftet. Sie sassen so dicht wie die Borsten einer Bürste. Namentlich vorn standen sie so nahe aneinander, dass eine Urethralmündung mit dem Auge nicht zu entdecken war. Die Wucherungen reichten über $5 \mathrm{Cm}$. nach oben und war bei gewaltsamem, iibrigens nicht besonders schmexzhaften Vordringen des Fingers dicht über ihnen die Portio zu fühlen. Eine specielle Behandlung war unnöthig und unmöglich.

Mit Sicherheit war diese papillöse Degeneration der Vaginalschleimhaut auf den langjährigem, von der Gonorrhoe herstammenden, nie behandelten Katarrh zu beziehen. Trotzdem war niemals, soweit es sich nachträglich eruiren liess, ein Zeichen einer Erkrankung der Gebärmutter oder ihrer Umgebung vorhanden gewesen. Und behauptete Jemand, dass vielleicht doch einmal Perimetritis bestanden habe, so ist der Beweis geliefert, dass sie selbst ohne Behandlung heilen kann.

In beiden Fällen folgte der Infection Sterilität aus verschiedenen Gründen. Doch will ich an dieser Stelle ausdrücklich. bemerken, dass die Sterilität nicht eine nothwendige Consequenz 
eines Cervicalcatarrhs zu sein braucht. Ich habe einen Fall beobachtet, wo während florider Gonorrhoe des Mannes und der Frau Conception eintrat und die Schwangerschaft ihr glückliches Ende erreichte, ohne dass etwa Pelviperitonitis folgte. Fs scheinen hier überhaupt die chronischen, tiefere Veränderungen in den Geweben verursachenden Katarrhe, auch wenn die Secretion höchst gering ist, viel wichtiger resp. schädlicher zu. sein.

Habe ich nun durch die vorstehenden Fälle gezeigt, dass häufig die Gonorrhoe eine ungefährliche leicht heilende Krankheit ist, so will ich dureh die folgenden Fälle den Beweis führen, dass die Gonorrhoe eine sebr ernstliche Perimetritis im Gefolge haben kann.

6) Frau B. Der Ehemann kommt mit einem sehr stark secernirenden 'Tripper, der sich erst gestern eingestellt habe, zu mir. Er sagt, dass noch gestern der Coitus vollzogen sei, Seine Frat wäre im dritten Monat schwanger, fühle sich ganz gesund, und er wünsche zu wissen, ob es kein Präservativ gegen eine eventuelle Erkrankung der Frau gäbe.

Die Gonorrhoe des Mannes besserte sich bei der gewöhnlichen Behandlung. Eine Woche später, 9 Tage nach dem letzten Coitus, wurde ich zur Frau gerufen. Ich fand sie abortirend. Der ganze Leib war schmerzhaft, die Scheide glühend heiss, in ihr lag das ïbelriechende Ovulum. Es wurde entfernt und die Vagina sowie der Uterus ausgespült. Am anderen Morgen trat ein halbstündlicher Schitttelfrost ein.

Es wurden Chinin, Blutegel und Priessnitz'sche Umschläge verordnet, die Vagina gereinigt und die Behandlung in der üblichen Weise fortgefiilart. Die acuten Symptome wurden geringer. Allabendlich hielt silch das Fieber auf 39,5-40,2. Links neben dem Uterus war das herabgesunkene Ovarium deutlich zu fühlen. Es schien sehr rund und circa wallnussgross zu sein. Allmälig bildete sich hier ein Exsudat, welches hinten nach rechts bis iiber die Mittellinie ragte. Vom. Uterus war es durch eine Rinne abzugrenzen, von aussen konnte es schon wegen der Schmerzhaftigkeit nicht gefühlt werden. Wie ich nicht selten gerade bei derartigen Perimetritiden zu beobachten Gelegenleit hatte, traten auch hier von Zeit zu Zeit wahre Schmerzparoxysmen auf, welche bei recht sorgfältiger Untersuchung und Ausfragen nur auf die Ovarien bezogen werden können. Patientin kam durch Appetitlosigkeit und vierwöchentliches Fieber sehr herunter. Noch heute nach 8 Wochen kehrt manchmal eine Fieberexacerbation wieder und ist Patientin ans Bett gefesselt. Das Exsudat ist kaum kleiner geworden, die Menstruation ist höchst profus, und in der intermenstruellen Zeit besteht so reichlicher Eiterabgang, dass schon einige Mal an Durchbruch des Exsudats geglaubt wurde.

Eine so schwere Erkrankung ist kaum auf den Abort allein zan 
beziehen und es ist sicher, dass dieser Fall ein völliges Analogon zu den von Nöggerath mitgetheilten Krankengeschichten bietet. Nur erfolgte die Infection durch einen sehr floriden Tripper, und trotz der Heftigkeit der Erkrankung glaube ich die Prognose doch günstig stellen zu diurfen.

7) Frau F., kräftige Frau, hat mehrere Kinder gehabt, leidet seit 6 Wochen, ohne bewusste Veranlassung an sehr starkem Fluor und fortwährenden, quälenden Kreuzschmerzen, die so stark sind, dass Patientin nur ganz langsam mit vornübergebeugtem Körper zu gehen im Stande ist. $\mathrm{Zu}$ Anfang sei blutiger Schleim ausgeflossen, jetzt nur Eiter. Die letzte Menstruation, zu zeitig eingetreten, sei sehr profus gewesen und habe ungewöhnlich lange gedauert. Bei der Untersuchung zeigte sich die obere Partie der Vagina granulirt, wie bei einer bochschwangeren Primipara. Sonst fehlte jede entzïndliche Erscheinung. Die Portio hat keine Einrisse und ist scharfrandig, der Muttermund eng, der Cervicalkanal bildet eine weite Ampulle, in welche der Finger sich einbohren kann. Bei der Specularuntersuchung fliesst aus dem eben manuell dilatirten Muttermund eine grosse Menge Schleim und Eiter aus der Cervix. Der Uterus liegt so antevertirt, das der Fundus etwas unter dem oberen Rande der Symphyse sich befindet. Hakt man mit zwei Fingern hinter die Portio, so gelingt es nicht sie nach vorn zu ziehen. Unter heftigen Kreuzschmerzen federt sie gleichsam in ihre alte Position zurück.

Der Ehemann giebt zu, vor 4 Wochen einen Tripper gehabt zu haben. Bei der Untersuchung finden sich kleine Eiterflecken im Hemd, obwohl am Penis und Hoden nichts mehr nachzuweisen ist.

Patientin bekam ein grosses Exsudat, neben der linken Seite des Uterus. Der letztere wurde so nach rechts verschoben, dass man das Exsudat fast wie den Uterus in der Mitte fühlte. Neun Wochen lang lag Patientin im Bett. Dann trat unter Behandlung mit Jod - Jodkali - Glycerintampons eine entschiedene Besserung ein. Die vollständige Heilung wurde schliesslich in einem Soolbad erlangt. Jetzt, 10 Monate nach dem Beginn der Krankheit untersuchte ich Patientin wieder. Der Fluor war minimal, ein Exsudat oder eine Schmerzhaftigkeit in der Nähe war nicht mehr nachzuweisen. Patientin fühlt sich völlig gesund.

In den beiden letzten Fällen entstand die Perimetritis sicher in Folge der Infection mit Trippervirus. Sicher ist dies aber nicht das Gewöhnliche. Man wird die Perimetritis in dergleichen Fällen am besten als Analogon der Entzündung der Tunica vaginalis beim Manne auffassen dürfen. Auch ohne dass eine weder zu beweisende noch abzuleugnende Salpingitis besteht, ist die Perimetritis verständlich. Ist ja auch im Puerperium die fortschreitende Entzündung durch die Tuben der allerseltenste Fall. Schon ältere Autoren vergleichen die Oophoritis gonorrhoica mit 
der Epididymitis gonorrhoica. Wie die letztere bei einem gut behandelten Tripper fehlt und fast nur auf äussere traumatische Veranlassung hinzukommt, so wird auch die Perimetritis nicht die nothwendige, jedesmalige Folge eines virulenten Cervicallatarrhes zu sein brauchen.

Entgegen den Ansichten Vieler glaube ich behaupten zu können, dass der Cervicalkatarrh in der übergrossen Mehrzahl der Fälle mit Endometritis nicht complicirt ist. Dies lehrt vor allem die mikroskopische Untersuchung. Die Epithelformen, welche z. B. an einem aus dem Uterus gezogenen Laminariastab oder Pressschwamm haften, findet man höchst selten im Schleime der Cervix.

Zum Schlusse sei bemerkt, dass sicherlich auch Perimetritiden existiren, welche mit den Generationsvorgängen in gar keinem Zusammenhang stehen. Der Umstand, dass der Tripper eine häufige Erkrankung der Männer, die Perimetritis eine häufige der Weiber ist, berechtigt noch nicht zu dem Schlusse, dass immer ein Causalconnex bestehen müsse. Wenn heute ein Autor irgend ein anderes, dem Wesen der Dinge nach häufig existirendes aetiologisches Moment z. B. Erkältung, das Trauma des Coitus, Stuhiverstopfung a. s. w. aufstellte, so würde es nicht schwierig sein, beweisende Krankengeschichten zu sammeln.

Ich hoffe durch Vorstehendes den factischen Beweis geliefert zu haben, dass die Behauptungen Nöggerath's nicht durchaus richtig sind, und dass Schröder in seinem Lehrbuche diese Ansichten mit Recht als extravagant bezeichnet. 